\title{
Molecular classification of esophagogastric junction carcinoma correlated with prognosis
}

This article was published in the following Dove Press journal:

OncoTargets and Therapy

25 September 2017

Number of times this article has been viewed

\section{Long Zou ${ }^{1,2}$ \\ Yinying $\mathrm{Wu}^{\prime}$ \\ Ke $M a^{\prime}$ \\ Yangwei Fan' \\ Danfeng Dong' \\ Ningyan Geng ${ }^{2}$ \\ Enxiao $\mathrm{Li}^{\mathrm{l}}$}

'Department of Medical Oncology,

The First Affiliated Hospital of Xi'an Jiaotong University, Xi'an,

${ }^{2}$ Department of Gastroenterology,

Shangluo Central Hospital, Shangluo,

Shaanxi, People's Republic of China

Correspondence: Enxiao Li

Department of Medical Oncology,

The First Affiliated Hospital of Xi'an

Jiaotong University, Xi'an, 277 West

Yanta Road, Shaanxi 71006, People's

Republic of China

Tel +862985324038

Email doclienxiao@sina.com
Abstract: A novel molecular classification of gastric cancer by the Asian Cancer Research Group (ACRG) is a potential advance in diagnosis and treatment, and it helps to determine prognosis. The use of immunohistochemistry (IHC) rather than gene expression analysis to determine tumor subtypes was evaluated with the aim of determining the feasibility of using the ACRG molecular classification. A total of 69 esophagogastric junction (EGJ) carcinomas were classified as microsatellite instable (MSI, $17.40 \%, 12$ of 69), microsatellite stable with markers of epithelial-to-mesenchymal transition (MSS/EMT, 18.84\%, 13 of 69), microsatellite stable with active tumor protein 53 (MSS/TP53+, 27.53\%, 19 of 69), and microsatellite stable with inactive TP53 (MSS/TP53-, 36.23\%, 25 of 69). The molecular classification did not significantly correlate with anyone of the clinicopathological characteristics of the EGJ carcinoma patients, including age, gender, depth of tumor invasion, the presence of lymph node metastasis, histologic grade, and p-TNM stage of the American Joint Committee on Cancer $(P>0.05)$. Kaplan-Meier survival analysis and log rank tests showed that molecular classification, histologic grade, p-TNM stage, and postoperative adjuvant chemotherapy were significantly associated with overall survival (OS; $P<0.05$ ). MSI tumors had the best overall prognosis followed by MSS/TP53 ${ }^{-}$and MSS/TP53 ${ }^{+}$. MSS/EMT tumors had the worst overall prognosis. Multivariate analysis revealed that histologic grade (hazard ratio $[\mathrm{HR}]=2.216,95 \% \mathrm{CI}=1.202-4.086)$, p-TNM stage $(\mathrm{HR}=2.216$, 95\% CI $=1.202-4.086$ ), and molecular subtype ( $\mathrm{HR}=2.216,95 \% \mathrm{CI}=1.202-4.086)$ were independently associated with OS. The preliminary results suggested that the ACRG molecular classification may be a valuable independent prognostic marker for EGJ carcinoma patients and could be performed by IHC analysis.

Keywords: molecular biology, gastroesophageal junction, microsatellite instable, MDM2, immunohistochemical staining, survival

\section{Introduction}

Gastric cancer (GC) is the fourth most common malignancy and the second leading cause of cancer-related deaths worldwide. ${ }^{1}$ Despite declines in incidence and mortality, GC remains a major contributor to the global cancer burden and cancer-related disability-adjusted life-years. In Western countries, $\sim 70 \%$ of GC patients die within 5 years of diagnosis. ${ }^{2,3}$ There are two anatomical forms of GC, noncardia or distal GC and cardia GC, also known as esophagogastric junction (EGJ) carcinoma. ${ }^{4}$ The incidence of EGJ carcinoma has been increasing, particularly in Western countries, ${ }^{5,6}$ where it has become a public health concern. The etiology and clinicopathological features of EGJ carcinoma differ from those of distal GC. It is usually diagnosed at a more advanced stage and has a worse prognosis, ${ }^{7,8}$ primarily because of a limited understanding of its molecular characteristics.

The existence of various subtypes, based on histopathology and anatomic site, ${ }^{9}$ gene expression, ${ }^{10-13}$ gene amplification, ${ }^{10,11,14}$ DNA methylation, ${ }^{10,11}$ and numerous 
cancer-related aberrations, ${ }^{10,11,13,15}$ reveals that $\mathrm{GC}$ is a heterogeneous, complex disease. An integrative genomic analysis of GC performed by the Asian Cancer Research Group (ACRG) provides a molecular classification that can be used to guide the development of targeted agents. ${ }^{10}$ The ACGR classification is based on a principal component analysis of expression data and a small predefined set of gene expression signatures considered relevant to GC biology. ${ }^{16-18}$ The classification includes four subtypes characterized by microsatellite-instable (MSI), microsatellite stable with markers of epithelial-to-mesenchymal transition (MSS/EMT), microsatellite stable with active tumor protein 53 (MSS/TP53 ${ }^{+}$), and microsatellite stable with inactive TP53 (MSS/TP53 ${ }^{-}$). To reduce the costs encountered in clinical practice, the ACRG recommends the use of immunohistochemistry (IHC) and RNA in situ hybridization for tumor classification rather than gene expression assays. The MSI group can be identified by MutL protein homolog 1 (MLH1) assay, the MSS/EMT group can be identified by assay of E-cadherin (CDH1) expression, and the MSS/TP53 tumors can be identified by assays of mouse double minute 2 homolog (MDM2) and cyclin-dependent kinase inhibitor 1A (CDKN1A, alternatively P21) expression. ${ }^{10}$

The four subtypes are linked to distinct patterns of molecular differences, disease progression, and prognosis. This novel molecular classification of GC may spur translational research to improve diagnosis and treatment approaching precision medicine ${ }^{19}$ and help to determine prognosis and to customize treatment. In this study, we assayed MLH1, E-cadherin, MDM2, and P21 in 69 EGJ carcinoma patients by IHC and classified their carcinomas following the ACRG molecular criteria. The relationships of the molecular subtypes, clinicopathological characteristics, and prognosis of the EGJ patients were evaluated.

\section{Materials and methods \\ Collection of clinical samples}

The patients who underwent surgical resection and were pathologically diagnosed with EGJ carcinoma at the First Affiliated Hospital of Xi'an Jiaotong University (Xi'an, People's Republic of China) between December 2010 and December 2012 were enrolled in this study. Patients with noncardia or distal GC and other organ primary malignant tumor were excluded from the study. Those whose information was incomplete were not in the range of analysis. The tumors were classified following the p-TNM staging system of the American Joint Committee on Cancer (AJCC). ${ }^{20}$ Survival was defined as the interval from diagnosis to the end of follow-up, and patients were followed up until death or study completion in March 2017. The study was approved by the Ethics Committees of the First Affiliated Hospital of Xi'an Jiaotong University. All the patients selected for our study were fully informed about our experiment protocols and signed an informed consent to participate in this study.

\section{Immunohistochemical staining}

Formalin-fixed, paraffin-embedded EGJ carcinoma tissue was cut into 4- $\mu \mathrm{m}$ serial sections. For antigen retrieval, the sections were heated in $10 \mathrm{mM} \mathrm{pH} 6.0$ citrate buffer in a microwave at high power for 8 minutes, followed by heating at midlow power for 13 minutes. Immunohistochemical staining was performed using a streptavidin-biotin peroxidase kit (SP-9001/9002; Zhongshan Golden Bridge Biotechnology, Beijing, People's Republic of China). Then, the sections were treated with $3 \%$ hydrogen peroxide in methanol for 10 minutes at room temperature followed by incubation with reagent A for 15 minutes at room temperature. They were then incubated overnight with primary antibodies against MLH1 (clone EPR3894, 1:100, ab92312), MDM2 (clone 2A10, 1:40, ab16895), P21 (clone EPR362, 1:100, ab109520), and E-cadherin (polyclonal, 1:50, 20874-1-AP) at $4^{\circ} \mathrm{C}$. After washing with $\mathrm{PBS}$, the sections were incubated with reagents $\mathrm{B}$ and $\mathrm{C}$ for 15 minutes each at $37^{\circ} \mathrm{C}$. Diaminobenzidine (ZLI-9018; Zhongshan Golden Bridge Biotechnology) was added according to the kit manufacturer's instructions. The sections were rinsed with tap water, counterstained with Harris' hematoxylin, and coverslipped. Then, they were observed and independently scored by two pathologists.

\section{Scoring methods and molecular classification}

All the sections were observed and independently scored by two pathologists in a double-blind manner. Using a high-power microscope, ten fields of vision were randomly selected from each slice, with 100 cells counted in each field. In cases in which there was disagreement between the two pathologists that impacted the categorization of a case as having positive or negative staining, the case was reviewed jointly until consensus was achieved. The Sinicrope scoring method ${ }^{21}$ was used to evaluate both the IHC staining intensity and the proportion of stained epithelial cells in each field. The scores were 0 for $\leq 5 \%$ stained cells, 1 for $6 \%-25 \%$ stained cells, 2 for $26 \%-50 \%$ stained cells, 3 for $51 \%-75 \%$ stained cells, and 4 for $>75 \%$ stained cells. Intensity was 0 for negative staining, 1 for weak staining, 2 for moderate staining, and 3 for strong staining. The overall immunostaining score 
for each tumor specimen was calculated by multiplying the percentage score by the intensity score. For MLH1 expression, a score of 0 indicated negative expression (ie, loss of MLHI) ${ }^{22,23}$ For MDM2, p21, and E-cadherin expression, a final score of 0-2 indicated negative expression and 3-12 indicated positive expression. Tumors with loss of MLHI expression were classified as the MSI subtype, and the remaining specimens were classified as MSS. Among the MSS specimens, tumors with negative E-cadherin expression were classified as MSS/EMT, and those with negative MDM2 expression and positive $\mathrm{P} 21$ expression were classified as the MSS/TP53+. Tumors with positive MDM2 expression and negative P21 expression were classified as MSS/TP53- ${ }^{10}$

\section{Statistical analysis}

Statistical analysis was performed using SPSS software, version 21.0 (SPPS, Inc., Chicago, IL, USA). Bivariate associations of categorical variables were evaluated using Pearson's $\chi^{2}$ test or Fisher's exact test as appropriate. KaplanMeier plots and log rank tests were used for survival analysis. Multivariate analyses were based on Cox proportional hazards regression models. All statistical tests were twosided, and statistical significances were defined as $P<0.05$.

\section{Results}

\section{Patient baseline characteristics}

A cohort of 69 patients, 56 men (81\%) and 13 women (19\%), with a median of 62 and a range of 38-81 years of age were included. All patients enrolled in the study received surgical treatment and were pathologically diagnosed with EGJ carcinoma; 57 patients were treated by radical resection of gastric cardia cancer, ten by total gastrectomy, one by radical resection of $\mathrm{GC}$, and one by omentectomy. All patients did not receive postoperative adjuvant radiotherapy and neoadjuvant therapy; 41 patients received postoperative adjuvant chemotherapy. There were no perioperative mortalities. According to the eighth edition of the AJCC Cancer Staging Manual, ${ }^{20} 15$ (21.7\%) patients were in Stage I/II, and 54 (of $69 ; 78.3 \%$ ) patients were in Stage III/IV; 36 (52.2\%) patients were diagnosed with moderately differentiated (histologic grade 2) tumors, and $33(47.8 \%)$ patients were diagnosed with poorly differentiated (histologic grade 3 ) tumors.

\section{Molecular EGJ carcinoma subtypes and their association with clinicopathological characteristics}

IHC staining revealed that MLH1, MDM2, and P21 were located in the nucleus, and E-cadherin was located on cell membrane or in cytoplasm of the EGJ cancer cells (Figure 1). The molecular subtypes and baseline patient clinicopathological characteristics are shown in Table 1. Of the 69 EGJ carcinomas, 12 (17.4\%) were MSI tumors, 13 (18.8\%) were MSS/EMT tumors, 25 (36.2\%) were MSS/ TP53- tumors, and 19 (27.5\%) were MSS/TP53+ tumors. There were no significant differences in age, gender, depth of tumor invasion, the presence of lymph node metastasis, histologic grade, p-TNM stages as well as whether these patients received adjuvant therapy in the four molecular grade groups $(P>0.05$; Table 1$)$.

\section{Molecular subtypes of EGJ carcinoma associated with overall survival (OS)}

The median follow-up was 36.3 (range 1.0-75.0) months, and the median OS was 36.3 months. The median and mean OS for each subgroup by histologic grade, p-TNM stage, postoperative adjuvant chemotherapy, and molecular subtype are shown in the Kaplan-Meier survival graphs, and the results of log-rank tests are shown in Table 2. Kaplan-Meier survival analysis showed that the patients with poor histologic grade, advanced AJCC p-TNM stage, and MSS/EMT molecular subtype and those who did not accept postoperative adjuvant chemotherapy had a worse prognosis (Figure 2). Further post hoc analysis showed that only patients with MSI subtype vs those with MSS/EMT subtype performed a significant different prognosis $(P=0.039)$. MSI tumors had the best overall prognosis, followed by MSS/TP53 ${ }^{-}$and MSS/TP53 ${ }^{+}$. Multivariate analysis (Table 3 ) revealed that histologic grade (hazard ratio $[\mathrm{HR}]=2.216,95 \% \mathrm{CI}=1.202-4.086$ ), p-TNM stage $(\mathrm{HR}=2.216,95 \% \mathrm{CI}=1.202-4.086)$, and molecular subtype $(\mathrm{HR}=2.216,95 \% \mathrm{CI}=1.202-4.086)$ were independently associated with the OS of EGJ carcinoma patients. The results revealed that molecular classification was an independent prognostic marker for EGJ carcinoma patients and that the ACRG subtype classification could be performed by IHC.

\section{Discussion}

GC is a highly heterogeneous disease with multiple subtypes, each with distinct biological properties. ${ }^{7-13}$ The molecular classifications of The Cancer Genome Atlas (TCGA) cohort, ie, Epstein-Barr virus ${ }^{+}$, MSI, genome stable, and chromosomal instability (CIN), ${ }^{11}$ and the ACRG cohort, ${ }^{10}$ ie, MSI, MSS/EMT, MSS/TP53 ${ }^{+}$, and MSS/TP53-, add to what is known of GC etiology and pathogenesis. The two molecular classifications probably reflect different underlying properties. When tumors from the original ACRG cohort were 

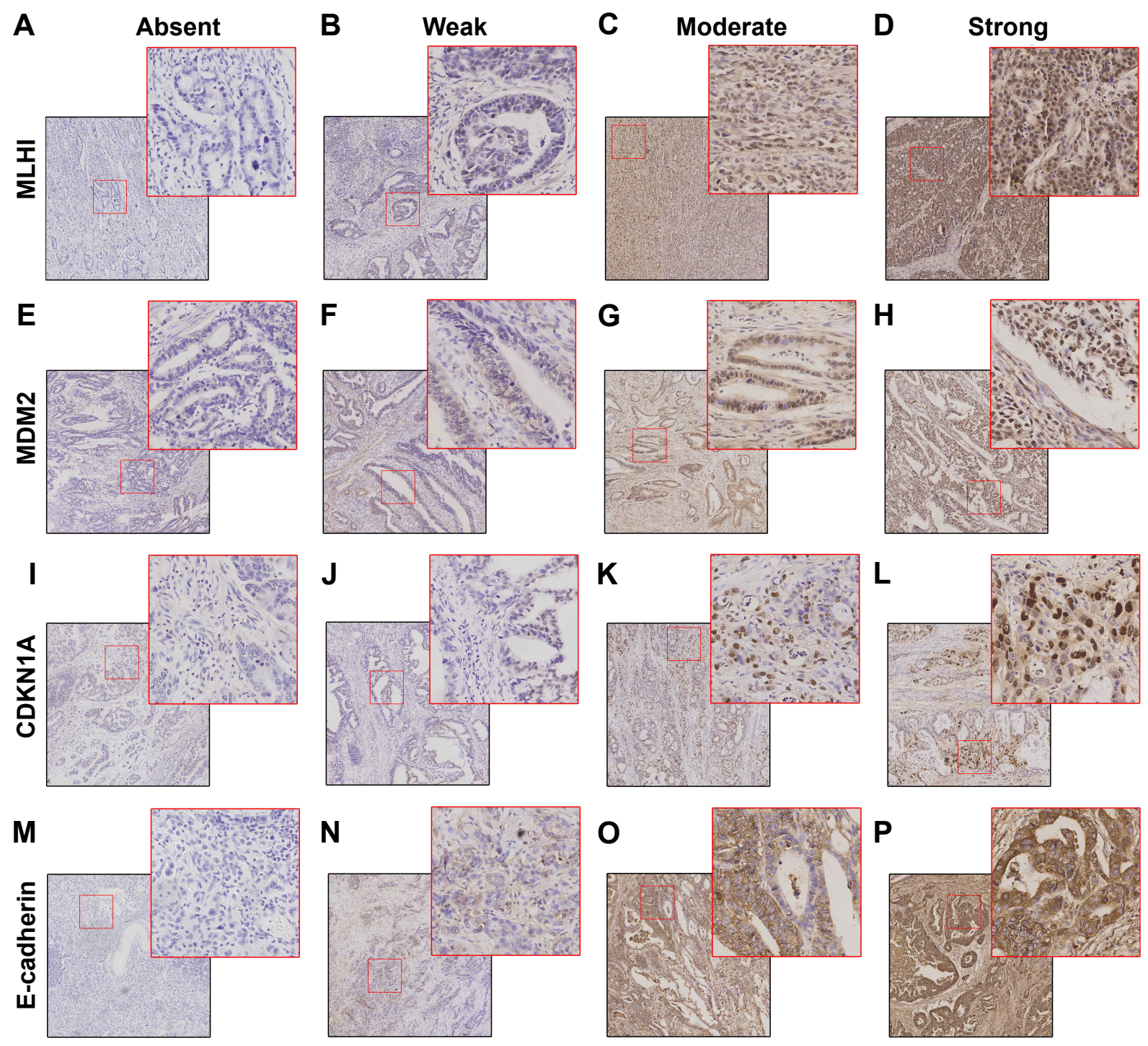

Figure I Representative images of multiple markers in EG] carcinoma.

Notes: (A-D) MLHI is completely absent, weak, moderate, and strong staining in EGJ carcinoma. (E-H) MDM2 is completely absent, weak, moderate, and strong staining in EGJ carcinoma. (I-L) CDKNIA is completely absent, weak, moderate, and strong staining in EGJ carcinoma. (M-P) E-cadherin is completely absent, weak, moderate, and strong staining in EGJ carcinoma. Black box, 100× magnification; red box, 400× magnification.

Abbreviations: CDKNIA, cyclin-dependent kinase inhibitor IA; EGJ, esophagogastric junction; MDM2, mouse double minute 2 homolog; MLHI, MutL protein homolog I.

classified using the TCGA genomic criteria, the association between molecular subtype and prognosis had decreased. ${ }^{10}$ In addition, CIN detection is complicated and requires indepth knowledge of the underlying mechanism. Gonzalez et $\mathrm{a}^{24}$ found that it was difficult to identify CIN subtype GC tumors with wild-type TP53 using IHC for p53 detection. In addition, the ACGR samples were from an Asian population, but the TCGA samples were from Europe and the USA. Because the ACRG molecular classification may be easier to use in clinical practice, especially in Chinese populations, we classified this series of 69 EGJ carcinoma patients using the four ACTRG molecular subtypes using IHC.
MSI is a genetic alteration consisting of the expansion or contraction of regions of microsatellites, which are repetitive nucleotide sequences caused by the inactivation of DNA mismatch repair genes (eg, MLH1 or MSH2). MSI has been reported in various tumors and can be detected by IHC assay of mismatch repair proteins or by profiling the Bethesda markers. ${ }^{25,26}$ It has the best overall prognosis and the lowest frequency of recurrence of the four subtypes. The prevalence of MSI in GC is estimated at $15 \%-30 \%$ of GC, and MSI-type tumors are more frequent in the antrum, in women and in older patients. ${ }^{10,11,27,28}$ In this cohort, $17.40 \%$ of the EGJ carcinomas were MSI, were the rarest subtype, 
Table I Four molecular subtypes and characteristics of $69 \mathrm{EGJ}$ carcinoma patients

\begin{tabular}{|c|c|c|c|c|c|}
\hline Characteristics & MSI & MSS/TP53 ${ }^{+}$ & MSS/TP53- & MSS/EMT & $P$-value \\
\hline Median age (years) & 57.50 & 61.00 & 64.00 & 63.00 & $0.583^{\mathrm{a}}$ \\
\hline Gender & & & & & 0.415 \\
\hline Male & $8(14.3 \%)$ & $15(26.8 \%)$ & 21 (37.5\%) & $12(21.4 \%)$ & \\
\hline Female & $4(30.8 \%)$ & $4(30.8 \%)$ & $4(30.8 \%)$ & I (7.6\%) & \\
\hline Depth of invasion & & & & & 0.558 \\
\hline $\mathrm{TI}-2$ & I (I2.5\%) & I (I2.5\%) & $5(62.6 \%)$ & I (I2.5\%) & \\
\hline T3-4 & II (18.0\%) & $18(29.5 \%)$ & $20(32.8 \%)$ & $12(19.7 \%)$ & \\
\hline Lymph node metastasis & & & & & 0.336 \\
\hline No & I (6.0\%) & $4(23.5 \%)$ & $9(52.9 \%)$ & $3(17.6 \%)$ & \\
\hline Yes & $\mathrm{II}(2 \mathrm{I} .2 \%)$ & $15(28.8 \%)$ & $16(30.8 \%)$ & $10(19.2 \%)$ & \\
\hline Histologic grade & & & & & 0.311 \\
\hline Moderate & 8 (22.2\%) & $12(33.3 \%)$ & II (30.6\%) & 5 (13.9\%) & \\
\hline Poor & $4(12.1 \%)$ & 7 (2I.2\%) & $14(42.4 \%)$ & $8(24.3 \%)$ & \\
\hline AJCC p-TNM stage & & & & & 0.387 \\
\hline $\mathrm{I} / \mathrm{II}$ & $2(13.3 \%)$ & $2(13.3 \%)$ & 8 (53.4\%) & $3(20.0 \%)$ & \\
\hline III/IV & $10(18.5 \%)$ & $17(31.5 \%)$ & 17 (31.5\%) & $10(18.5 \%)$ & \\
\hline Adjuvant therapy ${ }^{\mathrm{b}}$ & & & & & 0.175 \\
\hline No & $6(14.6 \%)$ & $10(24.4 \%)$ & $14(34.2 \%)$ & II (26.8\%) & \\
\hline Yes & $6(21.4 \%)$ & $9(32.1 \%)$ & II (39.3\%) & $2(7.2 \%)$ & \\
\hline
\end{tabular}

Notes: ane-way ANOVA test was used. For all other variables, $\chi^{2}$ test was used. ${ }^{b}$ Postoperative adjuvant chemotherapy. All patients enrolled in the study did not receive postoperative adjuvant radiotherapy or neoadjuvant therapy.

Abbreviations: AJCC, American Joint Committee on Cancer; ANOVA, analysis of variance; EGJ, esophagogastric junction; MSI, microsatellite instable; MSS/EMT, microsatellite stable with markers of epithelial-to-mesenchymal transition; MSS/TP53+, microsatellite stable with active tumor protein 53 , and MSS/TP53 ${ }^{-}$, microsatellite stable with inactive TP53.

Table 2 Univariate analysis of prognosis for $69 \mathrm{EGJ}$ carcinoma patients

\begin{tabular}{|c|c|c|c|}
\hline Variables & $\begin{array}{l}\text { Median OS } \\
\text { (months) }\end{array}$ & $\begin{array}{l}\text { Mean OS } \\
\text { (months) }\end{array}$ & $P$-value \\
\hline Gender & & & 0.439 \\
\hline Male vs Female & 39.00 vs 19.00 & 41.888 vs 32.523 & \\
\hline Age at diagnosis (years) & & & 0.347 \\
\hline$\leq 60$ vs $>60$ & 36.30 vs 36.00 & 43.532 vs 37.476 & \\
\hline Depth of invasion & & & 0.079 \\
\hline TI-2 vs T3-4 & NR vs 28.00 & 61.095 vs 37.396 & \\
\hline Lymph node metastasis & & & 0.120 \\
\hline No vs yes & NR vs 28.00 & 50.963 vs 36.283 & \\
\hline Grade & & & 0.015 \\
\hline Moderate vs Poor & 47.00 vs 13.00 & 49.980 vs 28.967 & \\
\hline AJCC TNM stage & & & 0.011 \\
\hline I/II vs III/IV & NR vs 21.80 & 59.900 vs 34.376 & \\
\hline Adjuvant therapy ${ }^{\mathrm{a}}$ & & & 0.046 \\
\hline No vs Yes & 28.00 vs 36.3 & 33.750 vs 48.593 & \\
\hline Molecular subtype & & & 0.042 \\
\hline $\mathrm{MSI}$ vs MSS/TP53+ vs & NR vs 36.00 & 53.808 vs 39.021 & \\
\hline \multirow[t]{2}{*}{ MSS/TP53- vs MSS/EMT } & vs 39.00 vs & vs 40.736 vs & \\
\hline & 13.00 & 23.192 & \\
\hline
\end{tabular}

Notes: aPostoperative adjuvant chemotherapy. All patients enrolled in the study did not receive postoperative adjuvant radiotherapy or neoadjuvant therapy. $P$-values are calculated by log rank test. Bold data indicate a statistically significant difference. Abbreviations: AJCC, American Joint Committee on Cancer; EGJ, esophagogastric junction; MSI, microsatellite instable; MSS/EMT, microsatellite stable with markers of epithelial-to-mesenchymal transition; MSS/TP53', microsatellite stable with active tumor protein 53, and MSS/TP53-, microsatellite stable with inactive TP53; NR, the median OS was not reached at the end of the observation; OS, overall survival. and had the best prognosis. This was consistent with the percentage of MSI in the original ACRG cohort (12.5\%), but in that cohort, MSS/EMT subtype was the least frequent. It is possible that using only MLH1 expression for typing may underestimate the prevalence of MSI because PMS2, MSH2, and MSH6 may also be deficient and independent of MLH1 expression. ${ }^{29}$

A small minority of GC cases are associated with a germline mutation in $\mathrm{CDHI},{ }^{30}$ which is downregulated in epithelial tumorigenesis and is categorized as a tumor suppressor gene. ${ }^{31}$ The loss or downregulation of E-cadherin is a characteristic of EMT. ${ }^{32}$ In this study, the MSS/EMT subtype occurred in $18.84 \%$ of the EGJ carcinomas and had the worst prognosis. Li et a ${ }^{33}$ reported that in diffuse-type GC, CDH1 mutation was associated with shortened survival, independent of disease stage. However, molecular analysis by next-generation sequencing and IHC showed that E-cadherin expression was not significantly associated with intestinaltype GC. ${ }^{34}$ The prognostic value of $\mathrm{CDH} 1$ and its potential as a candidate therapeutic target in $\mathrm{GC}$ deserve further study.

Next-generation sequencing and molecular profiling in GC found that TP53 was the most frequently mutated gene and that it contributed to the occurrence and development of GC. 10,11,29,34 

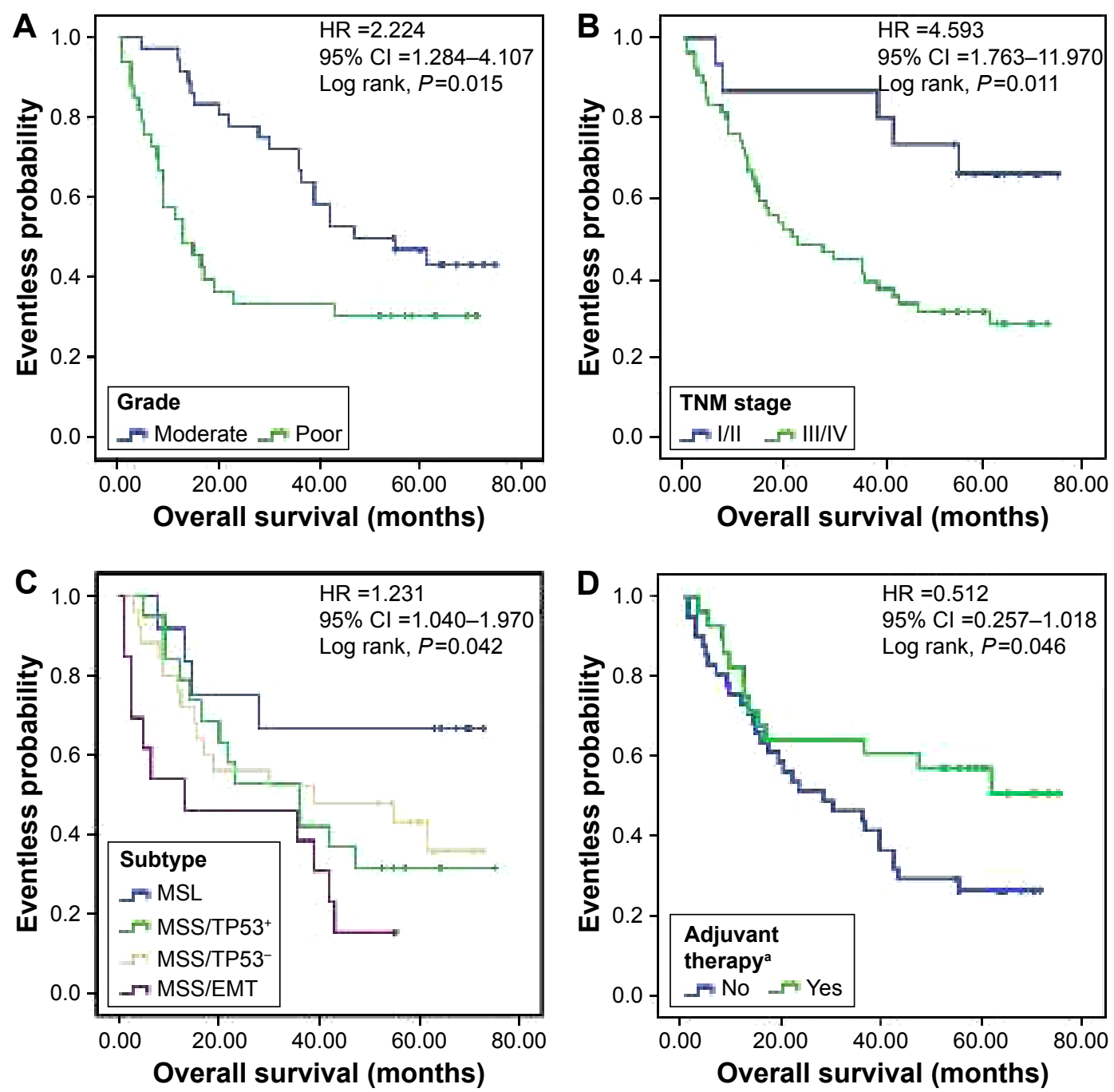

Figure 2 Kaplan-Meier survival curves for 69 EGJ carcinoma patients, grouped according to histologic grade (A), p-TNM stage (B), molecular subtypes (C), and postoperative adjuvant chemotherapy $($ D). HR and $95 \% \mathrm{Cl}$ were calculated by multivariable analysis after adjusting for several covariates (histologic grade, $\mathrm{P}$-TNM stage, molecular subtypes, and postoperative adjuvant chemotherapy).

Notes: adjuvant therapy. Postoperative adjuvant chemotherapy. All patients enrolled in the study did not receive postoperative adjuvant radiotherapy or neoadjuvant therapy. Abbreviations: EG], esophagogastric junction; HR, hazard ratio; MSI, microsatellite instable; MSS/EMT, microsatellite stable with markers of epithelial-to-mesenchymal transition; MSS/TP53 ${ }^{+}$, microsatellite stable with active tumor protein 53, and MSS/TP53-, microsatellite stable with inactive TP53.

Table 3 Multivariate analysis of prognosis for $69 \mathrm{EGJ}$ carcinoma patients

\begin{tabular}{|c|c|c|}
\hline Variables & HR (95\% Cl) & $P$-value \\
\hline Grade & & 0.011 \\
\hline Moderate vs Poor & $2.224(1.204-4.107)$ & \\
\hline AJCC TNM stage & & 0.002 \\
\hline I/II vs III/IV & $4.593(1.763-11.970)$ & \\
\hline Adjuvant therapy ${ }^{\mathrm{a}}$ & & 0.056 \\
\hline No vs Yes & $0.512(0.257-1.018)$ & \\
\hline Molecular subtype & & 0.028 \\
\hline $\begin{array}{l}\text { MSI vs MSS/TP53+ vs } \\
\text { MSS/TP53- vs MSS/EMT }\end{array}$ & I.43। (1.040-I.970) & \\
\hline
\end{tabular}

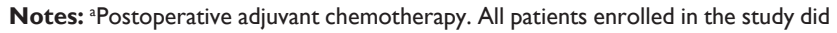
not receive postoperative adjuvant radiotherapy or neoadjuvant therapy. Bold data indicate a statistically significant difference.

Abbreviations: AJCC, American Joint Committee on Cancer; EGJ, esophagogastric junction; HR, hazard ratio; MSI, microsatellite instable; MSS/EMT, microsatellite stable with markers of epithelial-to-mesenchymal transition; MSS/TP53', microsatellite stable with active tumor protein 53 , and MSS/TP53-, microsatellite stable with inactive TP53.
Consequently, the ACRG stratified the remaining (non-MSI and non-EMT) tumors by the presence of TP53 activation. In both this cohort and the ACRG cohort, the non-MSI and non-MSS/EMT subtypes were the most common, comprising $63.76 \%$ and $62 \%$ of the total cases, respectively. The difference was that in the ACRG cohort, MSS/TP53 ${ }^{+}$was the most common subtype (14 of 32, 43.75\%) followed by MSS/TP53( 9 of $32,28.13 \%$ ), whereas in this cohort, MSS/TP53- was the most common (36.23\%), followed by MSS/TP53 $3^{+}(27.53 \%)$. In this cohort, the prognosis of MSS/TP53-, with a median OS of 39 months, was little better than that of MSS/TP53', which had a median OS of 36 months. In the ACRG cohort, MSS/TP53 ${ }^{+}$had a better prognosis than MSS/TP53-.

In the ACRG cohort study, GC molecular analysis was significantly associated with clinical phenotypes, such as 
age, grade, World Health Organization classification, AJCC stage, tumor invasion, and lymph node metastasis. ${ }^{10}$ In this study, the EGJ carcinoma molecular analysis did not correlate with those clinical variables. On the one hand, the difference may be a consequence of the small sample sizes or the different tumor types. On the other hand, it is the recurrence but not the depth of invasion and nodal status that correlate with molecular classification, but due to limited information about the local and distant recurrences, we failed to further do the work. To sum up, from the above results, we concluded that EGJ carcinoma and GC may have different molecular characteristics and that the ACRG molecular classification can be determined by IHC analysis, but the method needs to further be evaluated.

\section{Disclosure}

The authors report no conflicts of interest in this work.

\section{References}

1. Van Cutsem E, Sagaert X, Topal B, Haustermans K, Prenen H. Gastric cancer. Lancet. 2016;388(10060):2654-2664.

2. Soerjomataram I, Lortet-Tieulent J, Parkin DM, et al. Global burden of cancer in 2008: a systematic analysis of disability-adjusted life-years in 12 world regions. Lancet. 2012;380(9856):1840-1850.

3. Maleki SS, Rocken C. Chromosomal instability in gastric cancer biology. Neoplasia. 2017;19(5):412-420.

4. Colquhoun A, Arnold M, Ferlay J, Goodman KJ, Forman D, Soerjomataram I. Global patterns of cardia and non-cardia gastric cancer incidence in 2012. Gut. 2015;64(12):1881-1888.

5. Pohl H, Sirovich B, Welch HG. Esophageal Adenocarcinoma incidence: are we reaching the peak? Cancer Epidemiol Biomarkers prev. 2010;19(6):1468-1470.

6. Buas MF, Vaughan TL. Epidemiology and risk factors for gastroesophageal junction tumors: understanding the rising incidence of this disease. Semin Radiat Oncol. 2013;23(1):3-9.

7. Maeda H, Okabayashi T, Nishimori I, et al. Clinicopathologic features of adenocarcinoma at the gastric cardia: is it different from distal cancer of the stomach? J Am Coll Surg. 2008;206(2):306-310.

8. Liu K, Zhang W, Chen X, et al. Comparison on clinicopathological features and prognosis between esophagogastric junctional adenocarcinoma (Siewert II/III types) and distal gastric adenocarcinoma: retrospective cohort study, a single institution, high volume experience in China. Medicine (Baltimore). 2015;94(34):e1386.

9. Shah MA, Khanin R, Tang L, et al. Molecular classification of gastric cancer: a new paradigm. Clin Cancer Res. 2011;17(9):2693-2701.

10. Cristescu R, Lee J, Nebozhyn M, et al. Molecular analysis of gastric cancer identifies subtypes associated with distinct clinical outcomes. Nat Med. 2015;21(5):449-456.

11. Cancer Genome Atlas Research Network. Comprehensive molecular characterization of gastric adenocarcinoma. Nature. 2014;513(7517): 202-209.

12. Tay ST, Leong SH, Yu K, et al. A combined comparative genomic hybridization and expression microarray analysis of gastric cancer reveals novel molecular subtypes. Cancer Res. 2003;63(12):3309-3316.

13. Tan IB, Ivanova T, Lim KH, et al. Intrinsic subtypes of gastric cancer, based on gene expression pattern, predict survival and respond differently to chemotherapy. Gastroenterology. 2011;141(2):476-485, 485. e471-e411.
14. Deng N, Goh LK, Wang H, et al. A comprehensive survey of genomic alterations in gastric cancer reveals systematic patterns of molecular exclusivity and co-occurrence among distinct therapeutic targets. Gut. 2012;61(5):673-684.

15. Wang K, Yuen ST, Xu J, et al. Whole-genome sequencing and comprehensive molecular profiling identify new driver mutations in gastric cancer. Nat Genet. 2014;46(6):573-582.

16. Loboda A, Nebozhyn MV, Watters JW, et al. EMT is the dominant program in human colon cancer. BMC Med Genomics. 2011;4:9.

17. Cancer Genome Atlas Network. Comprehensive molecular characterization of human colon and rectal cancer. Nature. 2012;487(7407): 330-337.

18. Cancer Genome Atlas Network. Comprehensive molecular portraits of human breast tumours. Nature. 2012;490(7418):61-70.

19. Rocken C. Molecular classification of gastric cancer. Expert Rev Mol Diag. 2017;17(3):293-301.

20. Rice TW, Gress DM, Patil DT, Hofstetter WL, Kelsen DP, BlackstoneEH. Cancer of the esophagus and esophagogastric junction: major changes in the American Joint Committee on Cancer eighth edition cancer staging manual. CA Cancer J Clin. 2017;67(4):304-317.

21. Sinicrope FA, Ruan SB, Cleary KR, Stephens LC, Lee JJ, Levin B. bcl-2 and p53 oncoprotein expression during colorectal tumorigenesis. Cancer Res. 1995;55(2):237-241.

22. Thibodeau SN, French AJ, Roche PC, et al. Altered expression of hMSH2 and hMLH1 in tumors with microsatellite instability and genetic alterations in mismatch repair genes. Cancer Res. 1996;56(21): 4836-4840.

23. Thibodeau SN, French AJ, Cunningham JM, et al. Microsatellite instability in colorectal cancer: different mutator phenotypes and the principal involvement of hMLH1. Cancer Res. 1998;58(8): $1713-1718$

24. Gonzalez RS, Messing S, Tu X, McMahon LA, Whitney-Miller CL. Immunohistochemistry as a surrogate for molecular subtyping of gastric adenocarcinoma. Hum Pathol. 2016;56:16-21.

25. Dudley JC, Lin MT, Le DT, Eshleman JR. Microsatellite instability as a biomarker for PD-1 blockade. Clin Cancer Res. 2016;22(4): $813-820$.

26. Vilar E, Gruber SB. Microsatellite instability in colorectal cancer-the stable evidence. Nat Rev Clin Oncol. 2010;7(3):153-162.

27. Pedrazzani C, Corso G, Velho S, et al. Evidence of tumor microsatellite instability in gastric cancer with familial aggregation. Fam Cancer. 2009; 8(3):215-220.

28. Velho S, Fernandes MS, Leite M, Figueiredo C, Seruca R. Causes and consequences of microsatellite instability in gastric carcinogenesis. World J Gastroenterol. 2014;20(44):16433-16442.

29. Kim HS, Shin SJ, Beom SH, et al. Comprehensive expression profiles of gastric cancer molecular subtypes by immunohistochemistry: implications for individualized therapy. Oncotarget. 2016;7(28): 44608-44620.

30. Richards FM, McKee SA, Rajpar MH, et al. Germline E-cadherin gene (CDH1) mutations predispose to familial gastric cancer and colorectal cancer. Hum Mol Genet. 1999;8(4):607-610.

31. Cavallaro U, Christofori G. Cell adhesion and signalling by cadherins and Ig-CAMs in cancer. Nat Rev Cancer. 2004;4(2):118-132.

32. Bruner HC, Derksen PWB. Loss of E-cadherin-dependent cell-cell adhesion and the development and progression of cancer. Cold Spring Harb Perspect Biol. Epub 2017 May 15.

33. Li X, Wu WK, Xing R, et al. Distinct subtypes of gastric cancer defined by molecular characterization include novel mutational signatures with prognostic capability. Cancer Res. 2016;76(7):1724-1732.

34. Bria E, Pilotto S, Simbolo M, et al. Comprehensive molecular portrait using next generation sequencing of resected intestinal-type gastric cancer patients dichotomized according to prognosis. Sci Rep. 2016; 6:22982 


\section{Publish your work in this journal}

OncoTargets and Therapy is an international, peer-reviewed, open access journal focusing on the pathological basis of all cancers, potential targets for therapy and treatment protocols employed to improve the management of cancer patients. The journal also focuses on the impact of management programs and new therapeutic agents and protocols on

patient perspectives such as quality of life, adherence and satisfaction. The manuscript management system is completely online and includes a very quick and fair peer-review system, which is all easy to use. Visit http://www.dovepress.com/testimonials.php to read real quotes from published authors.

Submit your manuscript here: http://www.dovepress.com/oncotargets-and-therapy-journal 